\title{
Emission Spectroscopic Study on Gas-Gas Interactions in Glow Discharge Plasmas Using Several Binary Gas Mixtures
}

\author{
Kazuaki Wagatsuma \\ Institute for Materials Research, Tohoku University, 2-1-1 Katahira, Sendai 980-8577, Japan
}

\begin{abstract}
Emission spectra of constituent gas species from glow discharge plasmas using argon-helium, krypton-helium, argon-krypton, and krypton-argon gas mixtures were analyzed to elucidate collisional energy transfer between these gas species occurring in the plasma. In the argon-helium mixed gas plasma, the enhancement or quenching of particular Ar II lines was observed when helium was added to an argon-matrix glow discharge plasma, meaning that a redistribution in the population among the excited levels could be induced through argon-helium collisions. On the other hand, the krypton-helium plasma showed little change in the emission intensities of $\mathrm{Kr}$ II lines when helium was added to a krypton-matrix glow discharge plasma, meaning that energy exchanges between krypton and helium excited species occur inactively. These phenomena are principally because the excitation energy as well as the spin multiplicity between collision partners follow both the energy resonance conditions and the spin conservation rule in collisions of the second kind in the argon-helium system, but not in the krypton-helium system. In the argon-krypton and krypton-argon mixed gas plasmas, significant intensity changes of particular Ar II or Kr II lines could not be found; therefore, there were no dominant channels for energy exchanges between argon and krypton species in the mixed gas plasmas.
\end{abstract}

(Received December 7, 2009; Accepted January 25, 2010; Published March 10, 2010)

\section{Introduction}

Glow discharge optical emission spectrometry (GD-OES) is extensively employed for the direct analysis of solid samples, because the GD source has several benefits, such as rapid sampling through cathode sputtering, minimal sample pretreatment, and a wide concentration range in the quantification. The sampling process enables GD-OES to be well employed as a technique in surface analysis. Quantitative depth profiling of film-like samples now becomes the most important application in GD-OES, due to the simple experimental procedure without ultra-high vacuum conditions. Two books edited by Payling et al. ${ }^{1}$ and by Marcus and Broekaert ${ }^{2}$ have described the overall contents regarding GD-OES.

Unlike most of the other plasma sources, a GD plasma source is easily maintained without any instrumental modification when using various plasma gases, such as argon, neon, krypton, helium, and their mixtures. Although argon is usually used in conventional GD-OES, different gases have thus been investigated as an alternative plasma gas. ${ }^{3-9}$ These previous studies have reported that the kind of plasma gas dominantly determines the spectrum pattern of analyte elements: different spectral lines are excited depending on the plasma gas employed. ${ }^{3}$ The difference is not only in their relative intensities but in the kind of emission lines that can be excited for each plasma source. The characteristics should be considered when the measuring conditions and the plasma gas employed in GD-OES are selected for analyte elements of various samples. Penning ionization is the most important process for explaining the emission spectra from GD plasmas: ${ }^{10}$

E-mail: wagatuma@imr.tohoku.ac.jp
$\mathrm{M}^{0}+\mathrm{Ar}^{\mathrm{m}} \rightarrow \mathrm{M}^{+*}+\mathrm{Ar}^{\mathrm{g}}+\mathrm{e}^{-}$, where the superscripts $0, \mathrm{~m},+$ and $*$ mean a ground state, a metastable state, an ionic state, and an excited state, respectively. The $3 \mathrm{p}^{5} 4 \mathrm{~s}$ metastable states of the argon atom, the $3 \mathrm{p}^{5} 4 \mathrm{~s}^{3} \mathbf{P}_{0}(11.72 \mathrm{eV})$ and the $3 \mathrm{p}^{5} 4 \mathrm{~s}{ }^{3} \mathbf{P}_{2}$ $(11.55 \mathrm{eV}),{ }^{11}$ work as an energy donor through the collisions, contributing to the excitation of analyte atoms in GD-OES. In several elements, particular emission lines of the singly-ionized atom are selectively excited through another collision process; for instance, the $\mathrm{Cu}$ II 224.70-nm line has very high emission intensity in an argon GD plasma, which was first reported by Steers. ${ }^{12}$ This effect can be caused by selective excitation to a particular $3 d^{9} 4 p$ excited level of copper ion through a charge transfer collision between an $\mathrm{Ar}$ ion and a $\mathrm{Cu}$ atom. ${ }^{12}$ This type of collision is generally represented by the following equation: ${ }^{12}$ $\mathrm{M}^{0}+\mathrm{Ar}^{+} \rightarrow \mathrm{M}^{+*}+\mathrm{Ar}^{\mathrm{g}}+\Delta E$, where the superscripts $0,+$, and * mean a ground state, an ionic state, and an excited state, respectively, and $\Delta E$ means the energy difference before and after the collision. The charge transfer collision is most likely to take place when the surplus energy, $\Delta E$, is very small; in other words, an energy resonance is needed, ${ }^{13}$ meaning that the excited states to be selectively ionized/excited could be principally determined by the kind of the plasma gas, because the gas species have to provide the internal energy just corresponding to the sum of the excitation energy and the ionization potential. Therefore, an appropriate combination between a particular emission line and a plasma gas could improve the detection sensitivity in GD-OES, in the case where this resonance condition is fulfilled for the corresponding excited energy level. Our recent research has indicated that very intense emission lines of nickel are excited in a krypton GD plasma, rather than an argon plasma, which could be explained by the charge transfer collision to particular $3 \mathrm{~d}^{8} 4 \mathrm{p}$ excited levels of the nickel ion. ${ }^{14}$ 
In our previous work, a GD plasma using argon-helium mixed gas was suitable for the excitation to ionic emission lines requiring large excitation energies which did not appear in a pure argon plasma., ${ }^{45-17}$ This effect would be caused by a charge transfer collision with a helium ion which is produced through gas-gas collisions in the mixed gas plasma. Because helium is much less ionized than argon, due to the high ionization potential, and also because the sputtering yield is lower due to the small mass, the sampling amount of analytes is relatively small, which is not suitable for the analytical application. Therefore, an argon-helium mixed gas plasma instead of a pure helium plasma could be employed to keep good sputtering conditions. One should investigate collisional energy transfers between gas species, in the case of the mixed gas plasmas, in order that the gas composition as well as the discharge condition can be optimized for the analytical application. The emission lines of constituent gas species can give useful information on such collision processes. However, few spectroscopic studies have been conducted in GD plasmas using mixed plasma gases, although the gas collision process was suggested in an argon-helium mixed gas plasma. ${ }^{14}$ In the present work, the behavior of gas emission lines was observed and discussed when argon-helium, krypton-helium, argonkrypton, or krypton-argon was employed as the plasma gas. Furthermore, emission spectra of silver were compared between the GD plasmas using these gas mixtures, indicating that the argon-helium mixed gas plasma is the most suitable for the excitation of silver ion, which can be explained by energy transfer through collisions between the plasma gases.

\section{Experimental}

The Grimm-style glow discharge lamp ${ }^{18}$ employed here has been described in our previous papers. The excitation source was in-house made according to the original Grimm model. ${ }^{19}$ High-purity argon (99.99995\%), krypton (99.9995\%), and helium (99.9999\%) were employed as the plasma gas. The lamp was evacuated to less than 1.3 Pa by two oil rotary pumps, and then the plasma gases were introduced to flow continuously during the measurement. The matrix constituent gas in argon-helium, krypton-helium, or argon-krypton binary gas mixtures was first introduced at a fixed partial pressure, and subsequently the partial pressure of the guest gas was controlled. Flow control of the plasma gases was carried out with two sets of gas valves consisting of a ball (on/off) valve and a needle valve, which were inserted in each gas line. The pressure of the plasma gas was measured with a Pirani gauge, whose readings had been corrected for each gas, at the vacuum port of the lamp.

The emission spectra were measured on a Czerny-Turner mounting spectrometer (P-5200, Hitachi Corp., Japan) equipped with a photomultiplier (R-955, Hamamatsu Photonics, Japan). The discharge power was supplied with a dc power supply device (PAD 1L-0.2L, Kikusui Electronics Corp., Japan). All the measurements were conducted in a constant voltage mode. We prepared a pure nickel plate $(99.9 \%)$ for the analysis of gas emission lines and a pure silver plate $(99.9 \%)$ for elucidating the excitation phenomenon of analytes when using the mixed gas GD plasmas. It was polished with water-proof emery papers (Nos. 180-600) and then rinsed with ethanol. Before the measurement, pre-discharge was carried out for a few minutes to remove the surface contaminants.

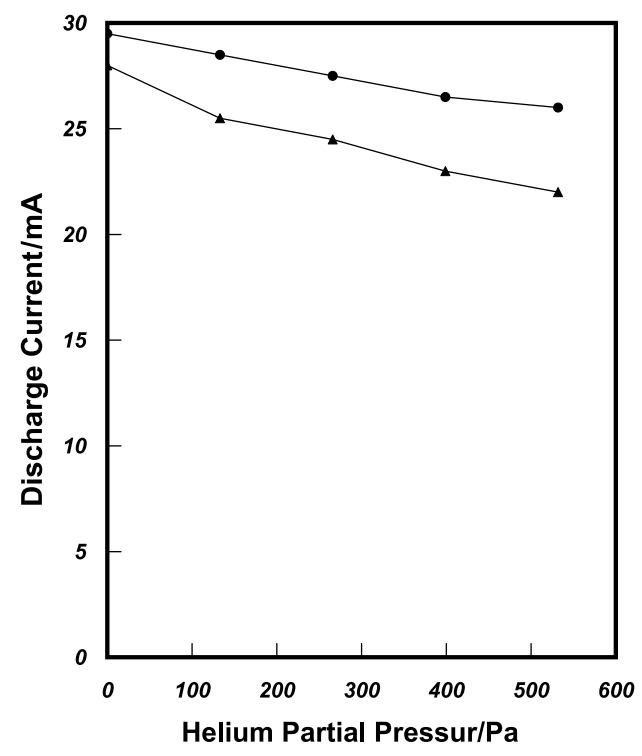

Fig. 1 Variations in the discharge current when helium is added to an argon plasma (triangle) and a krypton plasma (circle). Sample, pure nickel; gas pressure, $\mathrm{Ar}$ at $670 \mathrm{~Pa}$ or $\mathrm{Kr}$ at $670 \mathrm{~Pa}$ (fixed); discharge voltage, $600 \mathrm{~V}$ (fixed).

\section{Results and Discussion}

Argon-helium plasma

The emission characteristics of an argon-helium mixed gas plasma have been reported when copper, ${ }^{15}$ silver, ${ }^{16}$ and aluminum ${ }^{17}$ are employed as the analyte element, and also, emission lines of argon and helium have been investigated in an $\operatorname{argon}$ matrix $^{4}$ or a helium-matrix GD plasma. ${ }^{20}$ A variety of argon emission lines in an argon-helium plasma were re-measured in the present work, in order to investigate their excitation processes in more detail.

A large number of argon emission lines are observed from an argon GD plasma, and these lines originate from either singly-ionized (Ar II) or neutral argon species (Ar I) ${ }^{20}$ The Ar II lines are assigned to the optical transitions between several electron configurations of the argon ion; $3 p^{4} 4 p \rightarrow 3 p^{4} 4 s\left(3 p^{4} 3 d\right)$, $3 p^{4} 5 s\left(3 p^{4} 4 d\right) \rightarrow 3 p^{4} 4 p, \quad 3 p^{4} 4 f \rightarrow 3 p^{4} 3 d, \quad$ and so on, which comprise excited energy levels having doublet spin multiplicity (doublet line) or quartet spin multiplicity (quartet line). ${ }^{11}$ The excitation energies of the $3 p^{4} 4 p$ electron configuration are $19.68-21.50 \mathrm{eV}$ for the doublet and $19.22-19.97 \mathrm{eV}$ for the quartet, those of the $3 \mathrm{p}^{4} 5 \mathrm{~s}$ are $22.70-22.80 \mathrm{eV}$ for the doublet and $22.51-22.68 \mathrm{eV}$ for the quartet, and those of the $3 \mathrm{p}^{4} 4 \mathrm{~d}$ are $23.16-24.82 \mathrm{eV}$ for the doublet and $22.77-23.17 \mathrm{eV}$ for the quartet. ${ }^{11}$ We have reported a wavelength table of Ar II lines observed in the wavelength range of $350-500 \mathrm{~nm} .{ }^{19}$ On the other hand, the helium atom has several important electron configurations consisting of excited energy levels having singlet and triplet spin multiplicities, such as $1 \mathrm{~s} 2 \mathrm{~s}, 1 \mathrm{~s} 2 \mathrm{p}, 1 \mathrm{~s} 3 \mathrm{~s}, 1 \mathrm{~s} 3 \mathrm{p}$ excited states. The $1 \mathrm{~s} 2 \mathrm{~s}$ electron configuration produces two metastable energy levels: $1 \mathrm{~s} 2 \mathrm{~s}^{3} \mathbf{S}_{1}(19.82 \mathrm{eV})$ and $1 \mathrm{~s} 2 \mathrm{~s}{ }^{1} \mathbf{S}_{0}$ $(20.62 \mathrm{eV}) .{ }^{11}$ The excitation energies of the $1 \mathrm{~s} 2 \mathrm{p}$ electron configuration are $20.96 \mathrm{eV}$ for the singlet and $21.22 \mathrm{eV}$ for the triplet, those of the $1 \mathrm{~s} 3 \mathrm{~s}$ are $22.72 \mathrm{eV}$ for the singlet and $22.92 \mathrm{eV}$ for the triplet, and those of the $1 \mathrm{~s} 3 \mathrm{p}$ are $23.01 \mathrm{eV}$ for the singlet and $23.09 \mathrm{eV}$ for the triplet. ${ }^{11}$

Figure 1 shows variations in the discharge current as a function of the helium partial pressure added to an argon-matrix GD 


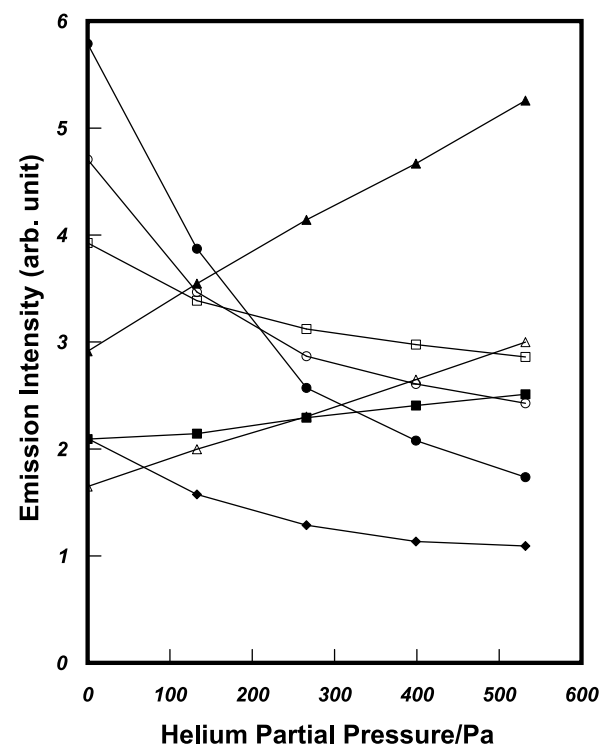

Fig. 2 Variation in the emission intensity of Ar II lines as a function of the helium partial pressure in an argon-helium mixed gas plasma. Argon lines: Ar II $465.80 \mathrm{~nm}$ (solid square), Ar II $460.96 \mathrm{~nm}$ (open square), Ar II $457.94 \mathrm{~nm}$ (open triangle), Ar II $454.52 \mathrm{~nm}$ (solid triangle), Ar II $410.39 \mathrm{~nm}$ (solid circle), Ar II $358.24 \mathrm{~nm}$ (open circle), and Ar II $358.17 \mathrm{~nm}$ (solid diamond). Sample, pure nickel; gas pressure, $\mathrm{Ar}$ at $670 \mathrm{~Pa}$ (fixed); discharge voltage, $600 \mathrm{~V}$ (fixed).

Table 1 Observed argon ionic lines and their assignment

\begin{tabular}{|c|c|c|}
\hline \multirow{2}{*}{ Wavelength/nm } & \multicolumn{2}{|c|}{ Assignment } \\
\hline & Upper level/eV & Lower level/eV \\
\hline Ar II 358.17 & $3 p^{4} 4 d^{4} \mathbf{F}_{3 / 2}(23.10)$ & $3 p^{4} 4 p^{4} \mathbf{D}_{1 / 2}$ \\
\hline Ar II 358.24 & $3 p^{4} 4 d^{4} \mathbf{F}_{5 / 2}(23.07)$ & $3 p^{4} 4 p^{4} \mathbf{D}_{3 / 2}(19.61)$ \\
\hline Ar II 410.39 & $3 \mathrm{p}^{4} 5 \mathrm{~s}{ }^{4} \mathbf{P}_{5 / 2}(22.52)$ & $3 \mathrm{p}^{4} 4 \mathrm{p}^{4} \mathbf{D}_{7 / 2}(19.49)$ \\
\hline Ar II 454.52 & $3 p^{4} 4 p^{2} \mathbf{P}_{3 / 2}(19.87)$ & $3 \mathrm{p}^{4} 4 \mathrm{~s}^{2} \mathbf{P}_{3 / 2}(17.14)$ \\
\hline Ar II 457.94 & $3 p^{4} 4 p^{2} \mathbf{S}_{1 / 2}(19.97)$ & $3 p^{4} 4 s^{2} \mathbf{P}_{1 / 2}(17.27)$ \\
\hline Ar II 460.96 & $3 p^{4} 4 p^{2} \mathbf{F}_{7 / 2}(21.14)$ & $3 \mathrm{p}^{4} 4 \mathrm{~s}^{2} \mathbf{D}_{5 / 2}(18.46)$ \\
\hline Ar II 465.80 & $3 \mathrm{p}^{4} 4 \mathrm{p}^{2} \mathbf{P}_{1 / 2}(19.80)$ & $3 \mathrm{p}^{4} 4 \mathrm{~s}^{2} \mathbf{P}_{3 / 2}(17.14)$ \\
\hline
\end{tabular}

plasma when the discharge is conducted at a fixed discharge voltage of $600 \mathrm{~V}$. The discharge current becomes gradually reduced with increasing the helium pressure; however, the change is small even when the amount of helium is equal to that of argon. This result implies that the helium addition causes little quenching of ionic argon species in the GD plasma, differing from an argon-nitrogen mixed gas plasma. ${ }^{21}$ Furthermore, in the case of a pure argon plasma, the discharge current becomes elevated when the gas pressure increases, which implies that the result of Fig. 1 is caused by any interaction between the argon and helium species.

Figure 2 shows variations in the emission intensities of several Ar II lines belonging to different assignment groups as a function of the helium partial pressure when helium is added to an argon GD plasma. In this case, the pressure of argon and the discharge voltage were fixed at $670 \mathrm{~Pa}$ and $600 \mathrm{~V}$, respectively. Their assignments are summarized in Table 1. The Ar II intensities are drastically changed by adding helium to the argon plasma, which implies that any collisional energy transfers occur between the argon and helium species. The intensities of three doublet Ar II lines having an excitation energy of

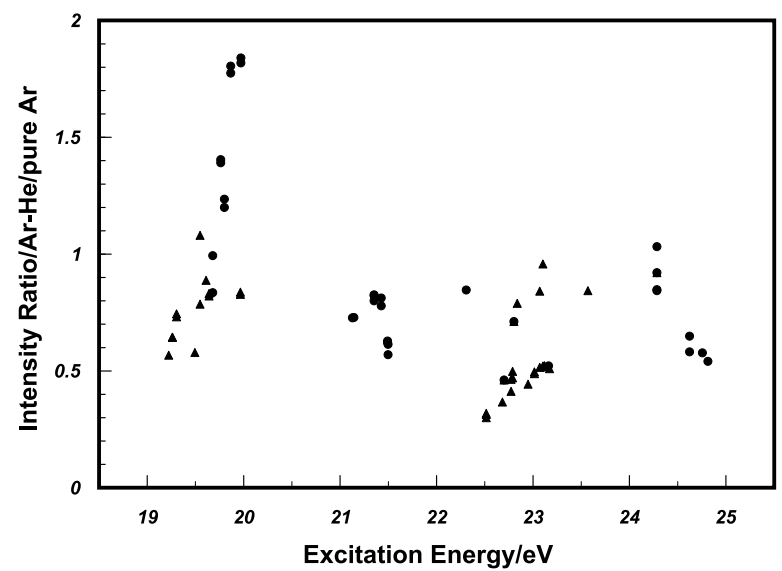

Fig. 3 Intensity ratios of Ar II doublet lines (circle) and quartet lines (triangle) between in an argon-helium plasma and in a pure argon plasma as function of their excitation energies. Sample, pure nickel; plasma gas composition, 670-Pa $\mathrm{Ar}+530-\mathrm{Pa} \mathrm{He} / 670-\mathrm{Pa} \mathrm{Ar}$; discharge voltage, $600 \mathrm{~V}$ (fixed).

19.8 - $20.0 \mathrm{eV}$ : Ar II $465.80 \mathrm{~nm}$, Ar II $454.52 \mathrm{~nm}$, and Ar II $457.94 \mathrm{~nm}$ are elevated, whereas those of quartet Ar II lines having larger excitation energies are drastically reduced by the helium addition. Figure 3 shows a plot of the intensity ratios of 67 Ar II emission lines versus their excitation energies, where the intensity ratio is estimated between in an argon-helium mixed gas plasma and in a pure argon plasma. The doublet lines (circle) and the quartet lines (triangle) are denoted with different markers. It is clearly found that only the doublet Ar II lines having an excitation energy of $c a .19 .9 \mathrm{eV}$ are selectively enhanced by the helium addition while the intensities of the other Ar II lines are generally reduced. Furthermore, Fig. 3 indicates that the quartet Ar II lines having excitation energies of $22.5-23.2 \mathrm{eV}$ are quenched more largely. This observation results from the increased or decreased population of the corresponding excited levels of the argon ion, which would be caused by any energy transfers between argon and helium species in the mixed gas plasma. It can also be considered from Figs. 1 and 2 that these enhancement/quenching phenomena are derived not from one-way quenching of ionic argon species but from any redistribution in the population among excited states of the argon ion caused by the helium addition.

One can consider various argon-helium collisions occurring in the GD plasma in order to explain changes in the population of the argon excited levels caused by adding helium gas. This type of collision is called a collision of the second kind. ${ }^{10}$ The probability of such a collision increases with decreasing the difference in the excitation energies between the corresponding energy levels; namely, a resonance condition in their excitation energies is required. ${ }^{13}$ In addition, according to Wigner's rule, the total spin of two collision partners should remain unchanged before and after the collision. ${ }^{10}$ If the total resulting spin is unchanged, the cross-section of the collisional energy transfer becomes large.

Because the ground state of the helium atom has a singlet term $\left({ }^{1} \mathbf{S}_{0}\right)$ and that of the argon ion a doublet term $\left({ }^{2} \mathbf{P}_{3 / 2}\right),{ }^{11}$ an energy-exchange collision between a doublet energy level of the argon ion and the helium singlet metastable $\left({ }^{1} \mathbf{S}_{0}\right)$ could occur more effectively than a collision with the helium triplet metastable $\left({ }^{3} \mathbf{S}_{1}\right)$ if the total spin is conserved. Equations (1) and (2) represent these collisions. 


$$
\begin{aligned}
& \operatorname{Ar}^{+*}(\text { doublet })+\mathrm{He}^{\mathrm{g}}\left(1 \mathrm{~s}^{2}{ }^{1} \mathbf{S}_{0}, 0.00 \mathrm{eV}\right) \longrightarrow \\
& \quad \operatorname{Ar}^{+\mathrm{g}}\left(3 \mathrm{p}^{5}{ }^{2} \mathbf{P}_{3 / 2}, 0.00 \mathrm{eV}\right)+\mathrm{He}^{*}(\text { singlet })+\Delta E, \\
& \mathrm{Ar}^{+*}(\text { doublet })+\mathrm{He}^{\mathrm{g}}\left(1 \mathrm{~s}^{2} \mathbf{S}_{0}, 0.00 \mathrm{eV}\right) \longrightarrow \\
& \quad \mathrm{Ar}^{+\mathrm{g}}\left(3 \mathrm{p}^{5}{ }^{2} \mathbf{P}_{3 / 2}, 0.00 \mathrm{eV}\right)+\mathrm{He}^{*}(\text { triplet })+\Delta E,
\end{aligned}
$$

where the superscripts $*$ and $\mathrm{g}$ indicate excited and ground states, respectively. The total spin remains unchanged in Eq. (1) whereas it is not conserved in Eq. (2). In the case of the quartet term system, a collision with the helium triplet metastable is more favorable for the energy exchange in argon-helium collisions, if the Wigner's rule is applied, as denoted in Eq. (3).

$$
\begin{aligned}
& \operatorname{Ar}^{+*}(\text { quartet })+\mathrm{He}^{\mathrm{g}}\left(1 \mathrm{~s}^{2}{ }^{1} \mathbf{S}_{0}, 0.00 \mathrm{eV}\right) \longrightarrow \\
& \quad \mathrm{Ar}^{+\mathrm{g}}\left(3 \mathrm{p}^{5}{ }^{2} \mathbf{P}_{3 / 2}, 0.00 \mathrm{eV}\right)+\mathrm{He}^{*}(\text { triplet })+\Delta E .
\end{aligned}
$$

As shown in Fig. 3, it seems that energy transfers from excited states of the argon ion to helium species are generally induced when helium is added to an argon GD plasma. For instance, the upper energy level $\left(3 \mathrm{p}^{4} 4 \mathrm{~d}^{4} \mathbf{F}_{3 / 2}, 23.10 \mathrm{eV}\right)$ of the Ar II $358.17 \mathrm{~nm}$, of which emission intensity is much reduced by the helium addition (see Fig. 2), could give its internal energy to the $1 \mathrm{~s} 3 \mathrm{p}^{3} \mathbf{P}$ excited state of the helium atom $(23.01 \mathrm{eV})$ through argon-helium collisions, which can fulfill the condition of Eq. (3). The following equation describes this reaction:

$$
\begin{aligned}
& \mathrm{Ar}^{+*}\left(3 \mathrm{p}^{4} 4 \mathrm{~d}^{4} \mathbf{F}_{3 / 2}, 23.10 \mathrm{eV}\right)+\mathrm{He}^{\mathrm{g}}\left(1 \mathrm{~s}^{2}{ }^{1} \mathbf{S}_{0}, 0.00 \mathrm{eV}\right) \longrightarrow \\
& \operatorname{Ar}^{+\mathrm{g}}\left(3 \mathrm{p}^{5}{ }^{2} \mathbf{P}_{3 / 2}, 0.00 \mathrm{eV}\right)+\mathrm{He}^{*}\left(1 \mathrm{~s} 3 \mathrm{p}{ }^{3} \mathbf{P}, 23.01 \mathrm{eV}\right)+\Delta E .
\end{aligned}
$$

Because the energy difference is very small $(\Delta E=0.09 \mathrm{eV})$, this reaction is expected to be effectively caused by the helium addition, resulting in a decreased population of the corresponding excited level of the argon ion and eventually decreasing the emission intensity.

On the other hand, in the argon-helium mixed gas system, there is an excitation condition in which the population of particular excited levels of the argon ion is elevated through argon-helium collisions. For instance, the emission intensity of the Ar II 457.94-nm line increases by adding helium to the argon GD plasma, as shown in Figs. 2 and 3. This effect is because the upper energy level $\left(3 p^{4} 4 p{ }^{2} \mathbf{S}_{1 / 2}, 19.97 \mathrm{eV}\right)$ can be more populated by energy transfer from the $1 \mathrm{~s} 2 \mathrm{~s}{ }^{1} \mathbf{S}_{0}$ excited level of the helium atom $(20.62 \mathrm{eV})$, which can fulfill the condition of Eq. (1). The following equation describes this reaction:

$$
\begin{aligned}
& \operatorname{Ar}^{+}\left(3 \mathrm{p}^{5}{ }^{2} \mathbf{P}_{3 / 2}, 0.00 \mathrm{eV}\right)+\mathrm{He}^{*}\left(1 \mathrm{~s} 2 \mathrm{~s}{ }^{1} \mathbf{S}_{0}, 20.62 \mathrm{eV}\right) \longrightarrow \\
& \operatorname{Ar}^{+*}\left(3 \mathrm{p}^{4} 4 \mathrm{p}^{2} \mathbf{S}_{1 / 2}, 19.97 \mathrm{eV}\right)+\mathrm{He}\left(1 \mathrm{~s}^{2}{ }^{2} \mathbf{S}_{0}, 0.00 \mathrm{eV}\right)+\Delta E .
\end{aligned}
$$

In this case, the major reason for this reaction is that the $1 \mathrm{~s} 2 \mathrm{~s}{ }^{1} \mathbf{S}_{0}$ excited level of the helium atom is a metastable state. Therefore, its population becomes larger compared to other excited levels of the helium atom, thus contributing to the excitation of the particular excited level of the argon ion which satisfies the spin conservation as well as the energy matching.

\section{Krypton-helium plasma}

The electron configuration of the krypton ion is similar to that of the argon ion, except that the outermost electrons of the krypton ion occupy the $\mathrm{N}$ shell, while those of the argon ion occupy the M shell. The Kr II lines are assigned to the optical transitions between several electron configurations of the krypton ion; $4 \mathrm{p}^{4} 5 \mathrm{p} \rightarrow 4 \mathrm{p}^{4} 5 \mathrm{~s}\left(4 \mathrm{p}^{4} 4 \mathrm{~d}\right), 4 \mathrm{p}^{4} 6 \mathrm{~s}\left(4 \mathrm{p}^{4} 5 \mathrm{~d}\right) \rightarrow 4 \mathrm{p}^{4} 5 \mathrm{p}$

\begin{tabular}{|c|c|c|}
\hline \multirow{2}{*}{ Wavelength/nm } & \multicolumn{2}{|c|}{ Assignment } \\
\hline & Upper level/eV & Lower level/eV \\
\hline Kr II 387.54 & $4 d^{4} 5 d^{2} \mathbf{F}_{5 / 2}(20.77)$ & $4 p^{4} 5 p^{4} \mathbf{S}_{3 / 2}(17.57)$ \\
\hline Kr II 392.02 & $4 p^{4} 5 d^{4} \mathbf{D}_{7 / 2}(20.00)$ & $4 p^{4} 5 p^{4} \mathbf{D}_{7 / 2}(16.83)$ \\
\hline Kr II 443.69 & $4 p^{4} 5 p^{2} \mathbf{P}_{3 / 2}(17.37)$ & $4 \mathrm{p}^{4} 5 \mathrm{~s}{ }^{4} \mathbf{P}_{1 / 2}(14.58)$ \\
\hline Kr II 483.21 & $4 p^{4} 5 p^{4} \mathbf{P}_{1 / 2}(16.88)$ & $4 \mathrm{p}^{4} 5 \mathrm{~s}{ }^{4} \mathbf{P}_{3 / 2}(14.27)$ \\
\hline
\end{tabular}
$4 \mathrm{p}^{4} 5 \mathrm{f} \rightarrow 4 \mathrm{p}^{4} 5 \mathrm{~s}$, and so on. ${ }^{22}$ As similar to the Ar II lines, they

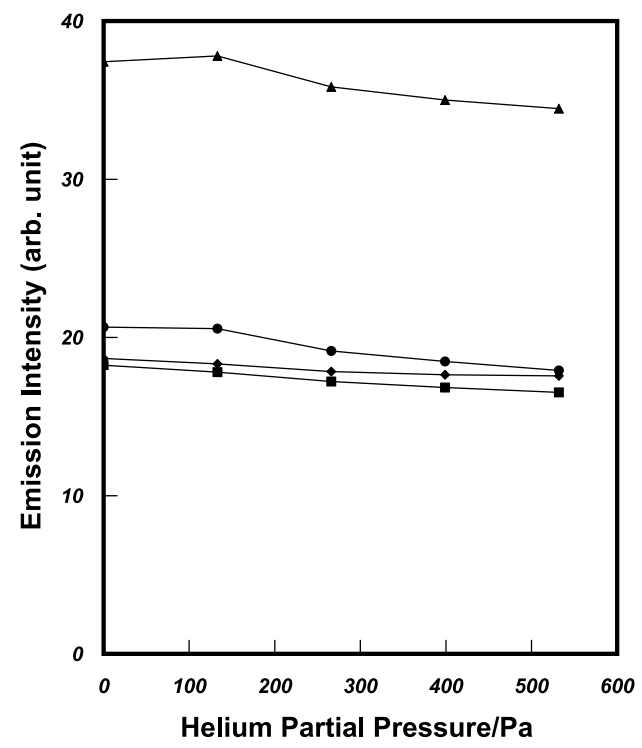

Fig. 4 Variation in the emission intensities of $\mathrm{Kr}$ II lines as a function of the helium partial pressure in a krypton-helium mixed gas plasma. Emission lines: Kr II $483.21 \mathrm{~nm}$ (square), $\mathrm{Kr}$ II $443.69 \mathrm{~nm}$ (diamond), $\mathrm{Kr}$ II $392.02 \mathrm{~nm}$ (triangle), and Kr II $387.54 \mathrm{~nm}$ (circle). Sample, pure nickel; gas pressure, $\mathrm{Kr}$ at $670 \mathrm{~Pa}$ (fixed); discharge voltage, $600 \mathrm{~V}$ (fixed).

Table 2 Observed krypton ionic lines and their assignment

comprise excited energy levels having doublet spin multiplicity (doublet line) or quartet spin multiplicity (quartet line). The excitation energies of the $3 p^{4} 5 p$ electron configuration are $17.25-18.88 \mathrm{eV}$ for the doublet and $16.60-17.57 \mathrm{eV}$ for the quartet, those of the $4 \mathrm{p}^{4} 6 \mathrm{~s}$ are $20.09-20.39 \mathrm{eV}$ for the doublet and $19.47-20.07 \mathrm{eV}$ for the quartet, and those of the $4 \mathrm{p}^{4} 4 \mathrm{~d}$ are $20.47-21.04 \mathrm{eV}$ for the doublet and $19.94-20.71 \mathrm{eV}$ for the quartet. ${ }^{22}$ It should be noted that their excitation energies are $2-3 \mathrm{eV}$ smaller than those of the corresponding excited levels of the argon ion.

A dependence of the discharge current on the helium partial pressure added to a krypton-matrix GD plasma is also shown in Fig. 1 when the discharge is conducted at a fixed discharge voltage of $600 \mathrm{~V}$. The discharge current is slightly reduced with increasing the helium pressure; however, the change is less than that of the argon-helium mixture even when large amounts of helium are mixed. It is thus considered that little interaction between krypton and helium species occurs in the GD plasma. Figure 4 shows variations in the emission intensities of several $\mathrm{Kr}$ II lines, whose transition assignments are listed in Table 2, as a function of the helium partial pressure when helium is added to a krypton GD plasma. In this case, a krypton pressure of $670 \mathrm{~Pa}$ and a discharge voltage of $600 \mathrm{~V}$ were employed. Differing from the argon-helium GD plasma, their emission intensities become slightly reduced by the helium addition 


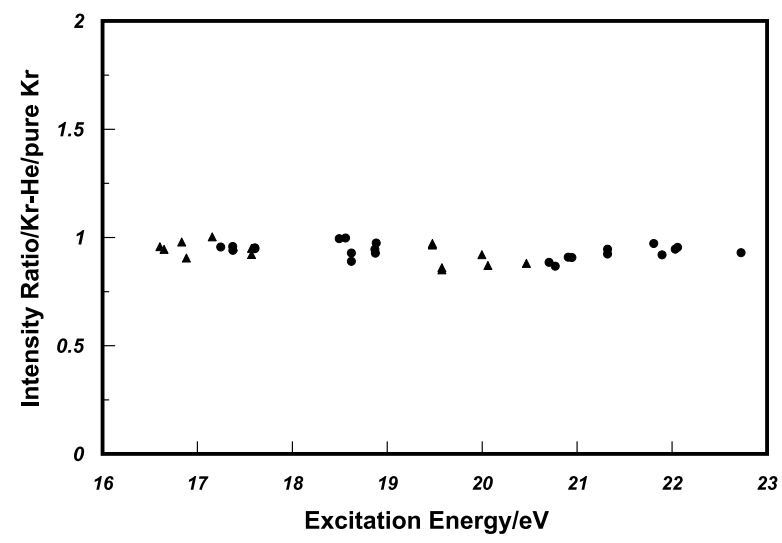

Fig. 5 Intensity ratios of $\mathrm{Kr}$ II doublet lines (circle) and quartet lines (triangle) between in a krypton-helium plasma and in a pure krypton plasma as function of their excitation energies. Sample, pure nickel; plasma gas composition, 670-Pa Kr + 530-Pa He/670-Pa Kr; discharge voltage, $600 \mathrm{~V}$ (fixed).

independent of the excitation energy as well as the spin multiplicity. The decrease in the $\mathrm{Kr}$ II intensities is probably due to the decrease in the discharge current, as shown in Fig. 1. Figure 5 shows a relationship between the intensity ratio of $40 \mathrm{Kr}$ II emission lines and their excitation energies, where the intensity ratio between in a krypton-helium mixed gas plasma and in a pure krypton plasma is estimated for each $\mathrm{Kr}$ II line. The composition of the mixed gas plasma is krypton of $670 \mathrm{~Pa}$ and helium of $530 \mathrm{~Pa}$. The doublet lines and the quartet lines are shown using circle and triangle markers, respectively. The intensity ratio of all the $\mathrm{Kr}$ II lines having doublet and quartet spin multiplicities becomes unchanged or slightly less than unity when adding helium to the krypton-matrix GD plasma, implying that few interactions between krypton and helium species occur in the plasma. One can find no selective enhancement/quenching of the $\mathrm{Kr}$ II emission intensities.

The collisional reactions followed by energy transfer, such as Eqs. (1) and (3), may also be caused in the krypton-helium mixed gas plasma, considering the experimental result in the argon-helium plasma. However, such reactions in the krypton-matrix plasma seem to be much less active than in the argon-matrix plasma. The major reason for this is mismatching in the excitation energy among excited energy levels of the krypton ion and the helium atom. For instance, one cannot find doublet energy levels having an excitation energy of $c a .20 \mathrm{eV}$ in Fig. 5, which could explain why no intensity enhancement of the $\mathrm{Kr}$ II lines around $20 \mathrm{eV}$ is observed in contrast to the argon-helium mixed gas plasma.

The difference in the energy transfer between argon-helium and krypton-helium collisions should exert an influence on the population in excited levels of helium species. Figure 6 shows variations in the emission intensity of the He I 388.87-nm line $\left(1 \mathrm{~s} 3 \mathrm{p}^{3} \mathbf{P}, 23.01 \mathrm{eV}\right)$ as a function of the helium partial pressure both in an argon-helium plasma and in a krypton-helium GD plasma. The intensities in the argon-helium plasma are 5-times larger than those in the krypton-helium plasma, indicating direct evidence that larger energy transfers to helium species are induced through argon-helium collisions compared to kryptonhelium collisions.

\section{Argon-krypton and krypton-argon plasmas}

An argon-krypton mixed gas plasma was investigated for

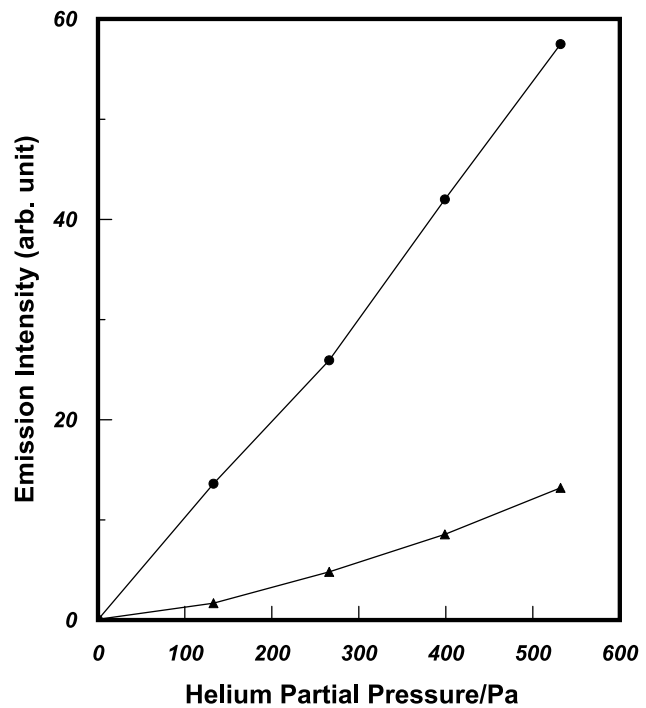

Fig. 6 Variation in the emission intensity of He I 388.87-nm line as a function of the helium partial pressure in an argon-helium mixed gas plasma (circle) or a krypton-helium mixed gas plasma (triangle). Sample, pure nickel; gas pressure, $\mathrm{Ar}$ or $\mathrm{Kr}$ at $670 \mathrm{~Pa}$ (fixed); discharge voltage, $600 \mathrm{~V}$ (fixed).

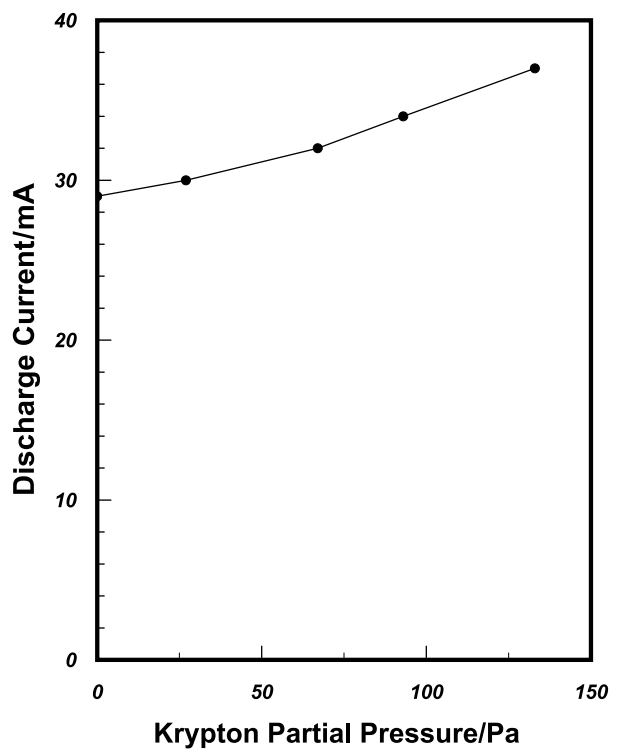

Fig. 7 Variations in the discharge current when krypton is added to an argon plasma. Sample, pure nickel; gas pressure, Ar at $670 \mathrm{~Pa}$ (fixed); discharge voltage, $600 \mathrm{~V}$ (fixed).

comparing the argon-helium and the krypton-helium plasmas. Figure 7 shows a variation in the discharge current as a function of the krypton partial pressure in an argon-matrix plasma. In this case, krypton gas was added up to $130 \mathrm{~Pa}$ while keeping the argon pressure to be $670 \mathrm{~Pa}$ at a discharge voltage of $600 \mathrm{~V}$. The addition of krypton raises the discharge current and, further, smaller amounts of krypton are effective for the current variation compared with the argon-helium mixed gas plasma (see Fig. 1). Figure 8 shows a plot of the intensity ratio of $65 \mathrm{Ar}$ II emission lines versus their excitation energies, where the ratio of the intensity in an argon-krypton mixed gas plasma to that in a pure argon plasma is estimated at a discharge voltage of $600 \mathrm{~V}$. 


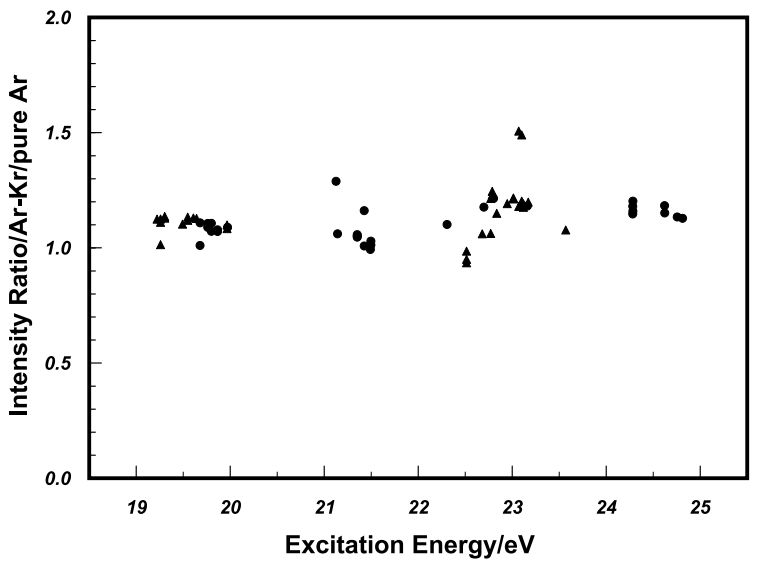

Fig. 8 Intensity ratios of Ar II doublet lines (circle) and quartet lines (triangle) between in an argon-krypton plasma and in a pure argon plasma as a function of their excitation energies. Sample, pure nickel; plasma gas composition, 670-Pa $\mathrm{Ar}+130-\mathrm{Pa} \mathrm{Kr} / 670-\mathrm{Pa} \mathrm{Ar}$; discharge voltage, $600 \mathrm{~V}$ (fixed).

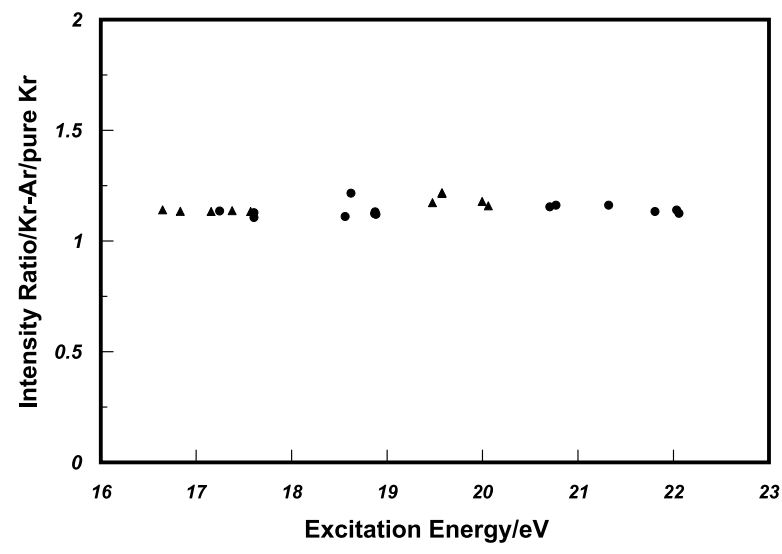

Fig. 9 Intensity ratios of $\mathrm{Kr}$ II doublet lines (circle) and quartet lines (triangle) between in a krypton-argon plasma and in a pure krypton plasma as function of their excitation energies. Sample, pure nickel; plasma gas composition, 670-Pa Kr + 130-Pa Ar/670-Pa Kr; discharge voltage, $600 \mathrm{~V}$ (fixed).

The plasma gas composition is argon of $670 \mathrm{~Pa}$ and krypton of $130 \mathrm{~Pa}$. The intensity ratios become more than unity by the krypton addition, meaning that their emission intensities increase in the argon-krypton mixed gas plasma. Furthermore, the intensity of the Ar II lines is generally elevated, regardless of the excitation energy as well as the spin multiplicity. This result would be derived from weak interactions between argon and krypton in this mixed gas plasma. Argon and krypton species could be excited/ionized individually, probably through electron collisions. The increase in the discharge current, as shown in Fig. 6, can be explained from the fact that krypton added to the argon-matrix plasma can be ionized additionally. This effect also explains the intensity increase of all the Ar II lines appearing in Fig. 7, because the addition of krypton makes the population of electrons to be elevated.

A krypton-argon mixed gas plasma was also investigated. Figure 9 shows a plot of the intensity ratio of $40 \mathrm{Kr}$ II emission lines versus their excitation energies, where the ratio of the intensity in a krypton-argon mixed gas plasma to that in a pure

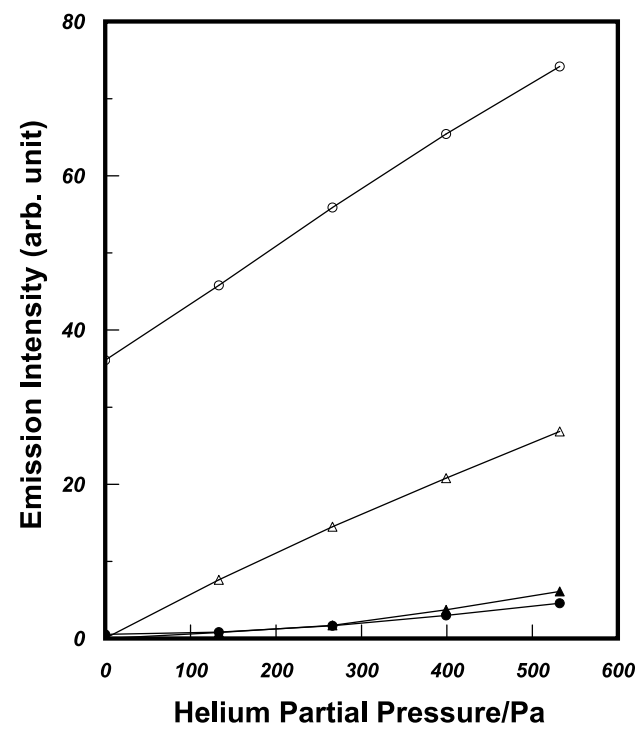

Fig. 10 Variation in the emission intensities of Ag II 228.00-nm line (circle) and Ag II 224.36-nm line (triangle) as a function of the helium partial pressure in an argon-helium mixed gas plasma (open markers) or a krypton-helium mixed gas plasma (solid markers). Sample, pure silver; gas pressure, $\mathrm{Ar}$ or $\mathrm{Kr}$ at $670 \mathrm{~Pa}$ (fixed); discharge voltage, $500 \mathrm{~V}$ (fixed).

krypton plasma is estimated at a discharge voltage of $600 \mathrm{~V}$. The plasma gas composition is krypton of $670 \mathrm{~Pa}$ and argon of $130 \mathrm{~Pa}$. The intensity ratios generally become $1.1-1.2$ by the argon addition independent of the excitation energy as well as the spin multiplicity, which is similar to the result of the argon-krypton plasma, as shown in Fig. 7. The reason for this is that krypton and argon species could be excited/ionized individually in the mixed gas plasma.

\section{Excitation of silver ion in helium-containing plasmas}

My previous study has reported that emission lines of singly-ionized silver (Ag II) are difficult to be excited using an argon GD plasma due to their large excitation energies. ${ }^{16}$ Especially, the $\mathrm{Ag}$ II lines resulting from the $4 d^{9} 5 d^{-}-4 d^{9} 5 p$ transition were observed only when the plasma gas contained helium. ${ }^{16,23}$ The $4 \mathrm{~d}^{9} 5 \mathrm{~d}$ excited levels can be populated through the charge transfer collision in which the helium ion is involved; therefore, the population of such excited species of helium should be concerned so that the ionic silver species can be effectively excited. Similarly, the $4 d^{9} 5 p$ excited levels would be populated through the Penning ionization where the helium metastable atom collides with the silver atom. Also in this case, the role of the excited helium species should be considered.

Figure 10 shows variations in the emission intensities of the Ag II $228.00 \mathrm{~nm}\left(4 \mathrm{~d}^{9} 5 \mathrm{p}{ }^{1} \mathbf{P}_{0}, 11.15 \mathrm{eV}\right)$ and the Ag II $224.36 \mathrm{~nm}$ $\left(4 \mathrm{~d}^{9} 5 \mathrm{~d}^{1} \mathbf{S}_{0}, 16.67 \mathrm{eV}\right)$ as a function of the helium partial pressure added to an argon-matrix plasma or a krypton-matrix plasma. One should notice that Ag II 228.00-nm line can be emitted from the pure argon plasma but not from the pure krypton plasma, whereas the Ag II 224.36-nm line cannot be excited from these pure gas plasmas. This effect would result from the following collision of the second kind between the argon metatstable atom and the silver ion:

$$
\begin{aligned}
& \mathrm{Ag}^{+\mathrm{g}}\left(4 \mathrm{~d}^{10}{ }^{1} \mathbf{S}_{0}, 0.00 \mathrm{eV}\right)+\mathrm{Ar}^{\mathrm{m}}\left(3 \mathrm{p}^{5} 4 \mathrm{~s}^{1} \mathbf{P}_{0}, 11.72 \mathrm{eV}\right) \longrightarrow \\
& \mathrm{Ag}^{+*}\left(4 \mathrm{~d}^{9} 5 \mathrm{p}^{1} \mathbf{P}_{0}, 11.15 \mathrm{eV}\right)+\operatorname{Ar}^{\mathrm{g}}\left(1 \mathrm{~s}^{2}{ }^{1} \mathbf{S}_{0}, 0.00 \mathrm{eV}\right)+\Delta E(6)
\end{aligned}
$$




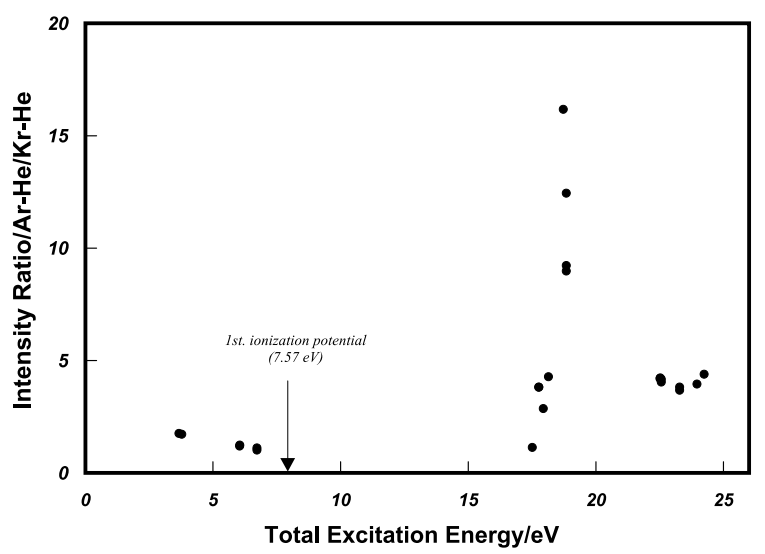

Fig. 11 Intensity ratios of $\mathrm{Ag} \mathrm{I}$ and $\mathrm{Ag}$ II lines between in an argon-helium plasma and in a krypton-helium plasma as function of their total excitation energies. Sample, pure silver; plasma gas composition, 670-Pa $\mathrm{Ar}+530-\mathrm{Pa} \mathrm{He} / 670-\mathrm{Pa} \mathrm{Kr}+530-\mathrm{Pa} \mathrm{He}$; discharge voltage, $500 \mathrm{~V}$ (fixed).

The metastable state of the krypton atom: $4 \mathrm{p}^{5} 5 \mathrm{~s}{ }^{3} \mathbf{P}_{0}(10.56 \mathrm{eV})$ and the $4 \mathrm{p}^{5} 5 \mathrm{~s}^{3} \mathbf{P}_{2}(9.92 \mathrm{eV})$, cannot provide enough energies to excite this excited level of the silver ion. In both of the Ag II lines, the addition of helium enhances the emission intensity of the silver ionic lines. Furthermore, the emission intensities increase more greatly in the argon-helium plasma rather than in the krypton-helium plasma, regardless of the helium amounts added. The ratio of the emission intensity in an argon-helium plasma to that in a krypton-helium for several silver atomic and ionic lines is plotted against the sum of the first ionization potential and their excitation energies, as shown in Fig. 11. As compared to the Ag I lines, the Ag II lines are generally enhanced in the argon-helium plasma, and especially, the intensity ratios become larger when the $\mathrm{Ag}$ II lines have a total excitation energy of $c a .19 \mathrm{eV}$. This ionization/excitation process is probably conducted through a Penning-type ionization; for example, that of the Ag II 228.00-nm is denoted as follows:

$$
\begin{gathered}
\operatorname{Ag}^{\mathrm{g}}\left(4 \mathrm{~d}^{10} 5 \mathrm{~s}^{2} \mathbf{S}_{1 / 2}, 0.00 \mathrm{eV}\right)+\mathrm{He}^{*}\left(1 \mathrm{~s} 2 \mathrm{~s}^{3} \mathbf{S}_{1}, 19.82 \mathrm{eV}\right) \longrightarrow \\
\mathrm{Ag}^{+*}\left(4 \mathrm{~d}^{9} 5 \mathrm{p}^{1} \mathbf{P}_{0}, 11.15 \mathrm{eV}\right)+\mathrm{He}^{\mathrm{g}}\left(1 \mathrm{~s}^{2}{ }^{1} \mathbf{S}_{0}, 0.00 \mathrm{eV}\right)+\mathrm{e}^{-}
\end{gathered}
$$

In this case, the population of the helium metastable determines this collision directly. Further, the charge transfer collision with the helium ion, whose internal energy is $24.5 \mathrm{eV}$, possibly populates the $4 d^{9} 5 d$ state requiring larger energies than the $4 d^{9} 5 p$ state of the silver ion. Larger amounts of such helium excited species can be produced in the argon-helium gas system because energy transfers from the argon to helium excited species occur more actively. Therefore, an argon-helium mixed gas is recommended for excitations of the Ag II lines.

\section{Conclusions}

In the argon-helium mixed gas plasma, the enhancement or quenching of particular Ar II lines is observed when helium was added to an argon-matrix glow discharge plasma, resulting in a redistribution in the population among the excited levels of the argon ion. The quenching of the argon excited levels occurs more dominantly, and thus the population of the helium excited species is elevated in the argon-helium plasma. This effect can be employed to produce helium excited species having a larger internal energy, which are suitable for excitations of ionic lines requiring large excitation energies such as ionic lines of silver. On the other hand, the krypton-helium plasma showed little change in the emission intensities of $\mathrm{Kr}$ II lines when helium was added to a krypton-matrix glow discharge plasma. These phenomena are well explained from good matching in the excitation energy as well as from the spin conservation rule in the collision of the second kind. In the argon-krypton and krypton-argon mixed gas plasmas, the emission intensity of Ar II or Kr II lines is slightly enhanced; however, significant intensity changes of particular lines can not be observed. It is thus considered that there are no dominant channels for energy exchanges between argon and krypton species in these mixed gas plasmas.

\section{References}

1. R. Payling, D. Jones, and A. Bengtson (ed.), "Glow Discharge Optical Emission Spectrometry”, 1997, John Wiley and Sons, Chichester.

2. R. K. Marcus and J. A. C. Broekaert (ed.), "Glow Discharge Plasmas in Analytical Spectroscopy", 2003, John Wiley and Sons, Chichester.

3. K. Wagatsuma and K. Hirokawa, Anal. Chem., 1985, 57, 2901.

4. K. Wagatsuma and K. Hirokawa, Spectrochim. Acta, Part B, 1987, 42, 523.

5. K. Wagatsuma and K. Hirokawa, Anal. Chem., 1988, 60, 702 .

6. W. Fischer, A. Nomads, and H. Nickel, J. Anal. At. Spectrom., 1994, 9, 375.

7. B. Fernandez, N. Bordel, C. Perez, R. Pereiro, and A. Sanz Medel, J. Anal. At. Spectrom., 2002, 17, 1549.

8. B. Lange, R. Matschat, and H. Kipphardt, Anal. Bioanal. Chem., 2007, 389, 7.

9. J. Munoz, M. S. Dimitrijevic, C. Yubero, and M. D. Calzada, Spectrochim. Acta, Part B, 2009, 64, 167.

10. A. von Engel, "Ionized Gases", 1965, Clarendon Press, London.

11. C. E. Moore, "Atomic Energy Levels", 1948, Vol. I, NBS Circular 467, Washington D.C.

12. E. B. M. Steers and R. J. Fielding, J. Anal. At. Spectrom., 1987, 2, 239.

13. O. S. Duffendach and J. G. Black, Phys. Rev., 1929, 34, 35.

14. K. Wagatsuma and H. Honda, Spectrochim. Acta, Part B, 2005, 60, 1538.

15. K. Wagatsuma and K. Hirokawa, Spectrochim. Acta, Part B, 1991, 46, 269.

16. K. Wagatsuma, Z. Phys., 1996, D37, 231.

17. K. Wagatsuma, J. Anal. At. Spectrom., 1996, 11, 957.

18. K. Wagatsuma and K. Hirokawa, Surf. Interface Anal., 1984, 6, 34.

19. W. Grimm, Spectrochim. Acta, Part B, 1968, 23, 443.

20. K. Wagatsuma and K. Hirokawa, Spectrochim. Acta, Part B, 1995, 50, 109.

21. K. Wagatsuma and K. Hirokawa, Spectrochim. Acta, Part B, 1988, 43, 213.

22. C. E. Moore, “Atomic Energy Levels”, 1952, Vol. III, NBS Circular 467, Washington D.C.

23. M. Tsukiji and K. Wagatsuma, Microchem. J., 2007, 87, 175. 